\title{
On the electrodynamics of moving bodies at low velocities ${ }^{1}$
}

\author{
M. de Montigny ${ }^{a, b}$ and G. Rousseaux ${ }^{c}$ \\ ${ }^{a}$ Campus Saint-Jean, University of Alberta \\ 8406 - 91 Street \\ Edmonton, Alberta, Canada T6C 4 G9 \\ ${ }^{b}$ Theoretical Physics Institute, University of Alberta \\ Edmonton, Alberta, Canada T6G 2J1 \\ ${ }^{c}$ Université de Nice Sophia-Antipolis, Institut Non-Linéaire de Nice, \\ INLN-UMR 6618 CNRS-UNICE, 1361 route des Lucioles, \\ 06560 Valbonne, France
}

\begin{abstract}
We discuss the seminal article in which Le Bellac and Lévy-Leblond have identified two Galilean limits of electromagnetism [1], and its modern implications. We use their results to point out some confusion in the literature and in the teaching of special relativity and electromagnetism. For instance, it is not widely recognized that there exist two well defined non-relativistic limits, so that researchers and teachers are likely to utilize an incoherent mixture of both. Recent works have shed a new light on the choice of gauge conditions in classical electromagnetism. We retrieve Le Bellac-Lévy-Leblond's results by examining orders of magnitudes, and then with a Lorentz-like manifestly covariant approach to Galilean covariance based on a 5-dimensional Minkowski manifold. We emphasize the Riemann-Lorenz approach based on the vector and scalar potentials as opposed to the Heaviside-Hertz formulation in terms of electromagnetic fields. We discuss various applications and experiments, such as in magnetohydrodynamics and electrohydrodynamics, quantum mechanics, superconductivity, continuous media, etc. Much of the current technology where waves are not taken into account, is actually based on Galilean electromagnetism.
\end{abstract}

Key words: Galilean covariance, special relativity, electromagnetism, four-potential.

\footnotetext{
${ }^{1}$ E-mail: montigny@phys.ualberta.ca, Germain.Rousseaux@inln.cnrs.fr
} 


\section{Introduction}

The purpose of this article is to emphasize the relevance of Galilean covariance in physics, even nowadays, about one hundred years after Lorentz, Poincaré and Einstein, then facing the apparent incompatibility between Galilean mechanics and the full set of Maxwell equations, have developed a theory that turned into special relativity [2]. Seventy years later, Le Bellac and Lévy-Leblond (LBLL) observed that there exist not only one, but two well-defined Galilean (that is, non-relativistic) limits of electromagnetism: the so-called 'magnetic' and 'electric' limits [1]. Although special relativity has superseded Galilean relativity when it comes to the description of high energy phenomena, there exists a wealth of low-energy systems, particularly in condensed matter physics and low-energy nuclear physics, where Galilean covariance should not be ignored.

We wish to point out hereafter some confusion which results from not recognizing appropriately the two Galilean limits of electromagnetism. This follows from inacurate definitions of non-relativistic covariance, which is why we emphasize at once that the definition of Galilean covariance employed henceforth in this paper rests on its compatibility with the Galilean transformations of space-time (Eq. (6), below). Examples of misleading, though well known, such text presentations are mentioned in [1], and there were many more since then. The fact that one should be careful when dealing with electrodynamics at low velocities has been illustrated, for instance, in Ref. [3]. Let us illustrate this point with a simple example. Under a Lorentz transformation with relative velocity $\mathbf{v}$, the electric and magnetic fields, in vacuum, become

$$
\begin{aligned}
& \mathbf{E}^{\prime}=\gamma(\mathbf{E}+\mathbf{v} \times \mathbf{B})+(1-\gamma) \frac{\mathbf{v}(\mathbf{v} \cdot \mathbf{E})}{\mathbf{v}^{2}}, \\
& \mathbf{B}^{\prime}=\gamma\left(\mathbf{B}-\frac{1}{c^{2}} \mathbf{v} \times \mathbf{E}\right)+(1-\gamma) \frac{\mathbf{v}(\mathbf{v} \cdot \mathbf{B})}{\mathbf{v}^{2}},
\end{aligned}
$$

respectively. The fact that Galilean covariance is a much more subtle concept than simply taking the $v<<c$, or $\gamma \simeq 1$, limit is illustrated by the fact that Eq. (1) then becomes:

$$
\begin{aligned}
& \mathbf{E}^{\prime}=\mathbf{E}+\mathbf{v} \times \mathbf{B}, \\
& \mathbf{B}^{\prime}=\mathbf{B}-\frac{1}{c^{2}} \mathbf{v} \times \mathbf{E},
\end{aligned}
$$

which not only is not compatible with Galilean relativity but, worse, does not even satisfy the composition properties of transformation groups $[1,3]$. That is to say, a sequence of such transformations does not have the same form as above.

We have organized this article as follows. In Section 2, we recall the main results of LBLL [1] for later reference. In Section 3, we obtain these results using two arguments: one based on orders of magnitudes and a recent covariant approach with which the Galilean space-time is embedded into a five-dimensional space. Throughout the paper, we favour the Riemann-Lorenz formulation of electrodynamics, based on the scalar and vector potentials, over the Heaviside-Hertz approach which involved electromagnetic fields [4]. Discussion and applications are in Section 4. Therein

we present magnetohydrodynamics and electrohydrodynamics as two not-so-distant 
cousins which can be associated respectively with the 'magnetic' and 'electric' Galilean limits of electrodynamics. We show that engineers are used to employ these Galilean limits which they denote as the electro- and magnetoquasistatics. We revisited Feynman's proof of the magnetic limit and illustrate the latter within the realm of superconductivity. Finally, we reassess our current understanding of the electrodynamics of moving bodies by examining the Trouton-Noble experiment in a Galilean context...

\section{Galilean electromagnetism}

The purpose of LBLL was to write down the laws of electromagnetism in a form compatible with Galilean covariance rather than Lorentz covariance. As LBLL put it, such laws could have been devised by a physicist in the mid-nineteenth century [1]. Here, let us retrieve these laws from relativistic kinematics. The Lorentz transformation of a four-vector $\left(u^{0}, \mathbf{u}\right)$, where the four components have the same units, is given by (see chapter 7 of Ref. [5]):

$$
\begin{aligned}
& u^{\prime 0}=\gamma\left(u^{0}-\frac{1}{c} \mathbf{v} \cdot \mathbf{u}\right) \\
& \mathbf{u}^{\prime}=\mathbf{u}-\gamma \frac{\mathbf{v}}{c} u^{0}+(\gamma-1) \frac{\mathbf{v}}{\mathbf{v}^{2}} \mathbf{v} \cdot \mathbf{u}
\end{aligned}
$$

where $\gamma \equiv \frac{1}{\sqrt{1-\mathbf{v}^{2} / c^{2}}}$, with a relative velocity $\mathbf{v}$. The speed of light in the vacuum is denoted $c$. LBLL were the first to observe that this transformation admits two well-defined Galilean limits [1]. One limit is for timelike vectors:

$$
\begin{aligned}
& u^{\prime 0}=u^{0}, \\
& \mathbf{u}^{\prime}=\mathbf{u}-\frac{1}{c} \mathbf{v} u^{0},
\end{aligned}
$$

which, as we shall see, may be related to the so-called electric limit. The second limit is for spacelike vectors:

$$
\begin{aligned}
& u^{\prime 0}=u^{0}-\frac{1}{c} \mathbf{v} \cdot \mathbf{u}, \\
& \mathbf{u}^{\prime}=\mathbf{u},
\end{aligned}
$$

and will be associated to the magnetic limit. As it is well known, the space-time coordinates can be described by timelike vectors only. Indeed, Eq. (4) has the form of Galilean inertial space-time transformations:

$$
\begin{aligned}
& \mathbf{x}^{\prime}=\mathbf{x}-\mathbf{v} t \\
& t^{\prime}=t
\end{aligned}
$$

Nevertheless, other vectors, such as the four-potential and four-current, may transform as one or the other of the two limits.

An example of the subtlety of non-relativistic kinematical covariance is that it is quite common to neglect to enforce the condition that a non-relativistic limit involves not only low-velocity phenomena, but also large timelike intervals then one obtains different kinematics, referred to as Carroll kinematics [6]. In other terms, a Galilean 
world is one within which units of time are naturally much larger than units of space. The existence of events physically connected by large spacelike intervals would imply loss of causality, among other things. Other such kinematics, each one being some limit of the de Sitter kinematics, have been classified in [7].

The situation is similar with electric and magnetic fields. One needs to compare the module of the electric field $E$ to $c$ times the module of the magnetic field, i.e. $c B$. When the magnetic field is dominant, Eq. (1) reduces to a transformation referred to as the magnetic limit of electromagnetism:

$$
\begin{aligned}
& \mathbf{E}_{m}^{\prime}=\mathbf{E}_{m}+\mathbf{v} \times \mathbf{B}_{m}, \quad E_{m}<<c B_{m}, \\
& \mathbf{B}_{m}^{\prime}=\mathbf{B}_{m} .
\end{aligned}
$$

The other alternative, where the electric field is dominant, leads to the electric limit:

$$
\begin{aligned}
& \mathbf{E}_{e}^{\prime}=\mathbf{E}_{e}, \quad E_{e}>>c B_{e}, \\
& \mathbf{B}_{e}^{\prime}=\mathbf{B}_{e}-\frac{1}{c^{2}} \mathbf{v} \times \mathbf{E}_{e} .
\end{aligned}
$$

Indeed, the approximations $E_{e} / c>>B_{e}$ and $v<<c$ together imply that $E_{e} / v>>$ $E_{e} / c>>B_{e}$ so that we take $E_{e}>>v B_{e}$ in Eq. (1). Such an analysis of orders of magnitude is described in the next section.

From the Galilean transformations of space-time, Eq. (6), we find

$$
\nabla^{\prime}=\nabla, \quad \partial_{t^{\prime}}=\partial_{t}+\mathbf{v} \cdot \nabla .
$$

The fields transformations in the magnetic limit of Eq. (7) are clearly compatible with the use of Eq. (9) together with the transformations of the four-potential $(V, \mathbf{A})$ :

$$
\begin{aligned}
& V_{m}^{\prime}=V_{m}-\mathbf{v} \cdot \mathbf{A}_{m}, \\
& \mathbf{A}_{m}^{\prime}=\mathbf{A}_{m},
\end{aligned}
$$

(note the similarity with Eq. (5)) where

$$
\mathbf{E}_{m}=-\nabla V_{m}-\partial_{t} \mathbf{A}_{m}, \quad \mathbf{B}_{m}=\nabla \times \mathbf{A}_{m} .
$$

Similarly, the electric limit of Eq. (8) may be obtained from Eq. (9) and the transformations of the four-potential:

$$
\begin{aligned}
& V_{e}^{\prime}=V_{e}, \\
& \mathbf{A}_{e}^{\prime}=\mathbf{A}_{e}-\frac{\mathbf{v}}{c^{2}} V_{e} .
\end{aligned}
$$

This equation is similar to Eq. (4). Now, however, the fields are related to the four-potential by

$$
\mathbf{E}_{e}=-\nabla V_{e}, \quad \mathbf{B}_{e}=\nabla \times \mathbf{A}_{e} .
$$

In parallel with the two possible sets of transformations of the four-potential, there are two ways to transform the four-current $(\rho, \mathbf{j})$. In the magnetic limit, it transforms like Eq. (5):

$$
\begin{aligned}
& \rho_{m}^{\prime}=\rho_{m}-\frac{1}{c^{2}} \mathbf{v} \cdot \mathbf{j}_{m}, \\
& \mathbf{j}_{m}^{\prime}=\mathbf{j}_{m},
\end{aligned}
$$


and the continuity equation then reads

$$
\nabla \cdot \mathbf{j}_{m}=0
$$

The appearance of an 'effective' charge density $\rho_{m}^{\prime}=\rho_{m}-\frac{1}{c^{2}} \mathbf{v} \cdot \mathbf{j}_{m}$ is certainly one of the salient feature of the magnetic limit. We will refer the interested reader to the following works which discussed the effect of this effective charge without pointing out its Galilean origin for most of them [8].

For the electric limit, it transforms like Eq. (4):

$$
\begin{aligned}
& \rho_{e}^{\prime}=\rho_{e} \\
& \mathbf{j}_{e}^{\prime}=\mathbf{j}_{e}-\mathbf{v} \rho_{e},
\end{aligned}
$$

and the continuity equation has its usual form:

$$
\nabla \cdot \mathbf{j}_{e}+\partial_{t} \rho_{e}=0
$$

Finally, Maxwell's equations,

$$
\begin{aligned}
\nabla \times \mathbf{E} & =-\partial_{t} \mathbf{B}, & & \text { Faraday } \\
\nabla \cdot \mathbf{B} & =0, & & \text { Thomson } \\
\nabla \times \mathbf{B} & =\mu_{0} \mathbf{j}+\frac{1}{c^{2}} \partial_{t} \mathbf{E}, & & \text { Ampere } \\
\nabla \cdot \mathbf{E} & =\frac{1}{\epsilon_{0}} \rho, & & \text { Gauss }
\end{aligned}
$$

reduce, in the Galilean limits, to two respective forms. As the field transformation laws themselves, this fact is not so obvious if one naively takes the limit $c \rightarrow \infty$. In the next section, we present an argument based on dimensional analysis and orders of magnitude. In Ref. [1], it was found that, in the electric limit, the Maxwell equations reduce to:

$$
\begin{aligned}
& \nabla \times \mathbf{E}_{e}=\mathbf{0} \\
& \nabla \cdot \mathbf{B}_{e}=0 \\
& \nabla \times \mathbf{B}_{e}-\frac{1}{c^{2}} \partial_{t} \mathbf{E}_{e}=\mu_{0} \mathbf{j}_{e} \\
& \nabla \cdot \mathbf{E}_{e}=\frac{1}{\epsilon_{0}} \rho_{e} .
\end{aligned}
$$

Clearly, the main difference with the relativistic Maxwell equations is that here the electric field has zero curl in Faraday's law. In the magnetic limit, the Maxwell equations become

$$
\begin{aligned}
\nabla \times \mathbf{E}_{m} & =-\partial_{t} \mathbf{B}_{m}, \\
\nabla \cdot \mathbf{B}_{m} & =0 \\
\nabla \times \mathbf{B}_{m} & =\mu_{0} \mathbf{j}_{m} \\
\nabla \cdot \mathbf{E}_{m} & =\frac{1}{\epsilon_{0}} \rho_{m} .
\end{aligned}
$$

The displacement current term is absent in Ampère's law. 


\section{$3 \quad$ Recent analyses}

\subsection{Orders of magnitude}

Many errors occur within low-velocity limits of relativistic theories when one naively replaces some quantities with zero, rather than carefully comparing various orders of magnitudes involved in the equations. As we shall show herafter, we do not require fanciful mathematical tools to retrieve the two Galilean limits of electromagnetism of LBLL. As discussed by one of us in Ref. [9], a careful dimensional analysis of the fields equations is sufficient for this purpose. Therein, it is argued that the electric and magnetic limits may be retrieved by a careful consideration of the order of magnitude of the dimensionless parameters:

$$
\varepsilon \equiv \frac{L}{c T}, \quad \text { and } \quad \xi \equiv \frac{j}{c \rho},
$$

where $L, T, j$ and $\rho$ represent the orders of magnitude of length, time, current density, and charge density, respectively. The Galilean kinematics considered hereafter corresponds to the quasistatic limit $\varepsilon<<1$. The other extreme, $\varepsilon>>1$, leads to the so-called Carroll kinematics [6]. The main undesirable feature of this kinematical structure is the loss of causality.

The electric or magnetic character of the Galilean limits of electromagnetism is determined by the behavior of the parameter $\xi$. From Gauss's law and Ampère's law, Eq. (18), we find $\frac{c B}{E} \simeq \frac{j}{\rho c}$, so that

$$
\frac{c B}{E}=\xi
$$

Using this result and Eqs. (7), (8), we find:

$$
\begin{array}{ll}
\xi>>1: & \text { magnetic limit, } \\
\xi<<1: & \text { electric limit. }
\end{array}
$$

Returning to Eq. (21), we see that the magnetic limit correspond to the approximation $j>>c \rho$, that is, the spacelike component is larger than the timelike component. This echoes the transformation in Eq. (5). Conversely, the electric limit correspond to the approximation $c \rho>>j$, so that the spacelike component now is much larger than the timelike component. This is analogue to Eq. (4).

From Maxwell displacement current term in the Ampère's law, Eq. (18), we find

$$
B \simeq \frac{v E}{c^{2}}
$$

where $v$ denotes the ratio of orders of magnitude $L / T$. Similarly, the magnetic induction term of Faraday's law gives

$$
E \simeq v B
$$


If we substitute this result into Eq. (23), we find that the displacement current term and the full Faraday's law are compatible only if $v \simeq c$, that is, in the Lorentz covariant regime. However, Eq. (23) cannot be obtained if we drop $\partial_{t} \mathbf{E}$ from Ampère's law, so that it is compatible with the first and third lines of Eq. (19), i.e. in the electric limit. On the other hand, Eq. (24) is compatible with lines one and three of Eq. (20), i.e. the magnetic limit, because it does not appear if we drop the magnetic induction term $\partial_{t} \mathbf{B}$ of Faraday's law in line three of Eq. (18).

Following the lines of Ref. [10]-[14], we use the Riemann-Lorenz formulation of electromagnetism, which relies on the potentials as the basic quantities, in order to retrieve the two Galilean limits. This is in opposition to the Heaviside-Hertz formulation, which is based on the magnetic and electric fields [10]-[12]. In terms of potentials, the equations of classical electromagnetism read

$$
\begin{gathered}
\nabla^{2} V-\frac{1}{c^{2}} \frac{\partial^{2} V}{\partial t^{2}}=-\frac{\rho}{\epsilon_{0}}, \quad \text { Riemann equations, } \\
\nabla^{2} \mathbf{A}-\frac{1}{c^{2}} \frac{\partial^{2} \mathbf{A}}{\partial t^{2}}=-\mu_{0} \mathbf{j}, \\
\nabla \cdot \mathbf{A}+\frac{1}{c^{2}} \frac{\partial V}{\partial t}=0, \quad \text { Lorenz equation, } \\
\frac{\mathrm{d}}{\mathrm{d} t}(m \mathbf{v}+q \mathbf{A})=-q \nabla(V-\mathbf{v} \cdot \mathbf{A}), \quad \text { Lorentz force. }
\end{gathered}
$$

They can be obtained from the Fermi Lagrangian [15]

$$
\mathcal{L}_{F}=\frac{1}{2} \epsilon_{0} c^{2} \partial_{\mu} A_{\nu} \partial^{\mu} A^{\nu}
$$

We consider the full Lagrangian consisting of a field and a matter part, that is $\mathcal{L}=\mathcal{L}_{F}+\mathcal{L}_{M}$. The Euler-Lagrange equation,

$$
\frac{\partial \mathcal{L}}{\partial A_{\mu}}-\partial_{\nu}\left[\frac{\partial \mathcal{L}}{\partial\left(\partial_{\nu} A_{\mu}\right)}\right]=0
$$

leads to the following equations of motion (using Eq. (26) $\partial_{\mu} A^{\mu}=0$ )

$$
\partial_{\nu} \partial^{\nu} A^{\mu}=-\mu_{0} j^{\mu}
$$

with

$$
-\mu_{0} j^{\mu}=\partial \mathcal{L}_{M} / \partial A_{\mu} .
$$

The quasistatic approximation, $\varepsilon<<1$, of Eq. (25) reads

$$
\nabla^{2} V \simeq-\frac{\rho}{\epsilon_{0}} \quad \text { and } \quad \nabla^{2} \mathbf{A} \simeq-\mu_{0} \mathbf{j}
$$

from which we can define a further dimensionless ratio, $\frac{c A}{V} \simeq \frac{j}{\rho c}$, so that

$$
\frac{c A}{V} \simeq \xi
$$


Once again, this echoes our comment following Eq. (22): in the magnetic limit, the spacelike quantity $c A$ is dominant, whereas in the electric limit, it is the timelike quantity $V$ which dominates.

If we compare the two terms of the Lorenz (not Lorentz [13]) gauge condition, Eq. (26), we find

$$
\frac{|\nabla \cdot \mathbf{A}|}{\partial_{t} V / c^{2}} \simeq \frac{c T}{L} \frac{c A}{V} \simeq \frac{\xi}{\varepsilon}
$$

In the quasistatic regime, $\varepsilon<<1$, we find therefore two possibilities. If $\xi<<1$, like $\varepsilon$, then we are in the electric limit, and the gauge condition is similar to the Lorenz condition:

$$
\nabla \cdot \mathbf{A}_{e}+\frac{1}{c^{2}} \partial_{t} V_{e}=0
$$

On the other hand, in the magnetic limit, $\xi>>1$, then we drop $\partial_{t} V$, so that we obtain the Coulomb gauge condition:

$$
\nabla \cdot \mathbf{A}_{m}=0 .
$$

Let us use the orders of magnitude for the four-potential components and obtain thereof their Galilean transformations in the magnetic limit, Eq. (10), and the electric limit, Eq. (12). From Eq. (3) with $u^{0}=V / c$ and $\mathbf{u}=\mathbf{A}$, we find that the scalar potential $V$ and the vector potential A transform, under a Lorentz transformation, as

$$
\begin{aligned}
& V^{\prime}=\gamma(V-\mathbf{v} \cdot \mathbf{A}), \\
& \mathbf{A}^{\prime}=\mathbf{A}-\gamma \frac{\mathbf{v}}{c^{2}} V+(\gamma-1) \frac{\mathbf{v}}{\mathbf{v}^{2}} \mathbf{v} \cdot \mathbf{A} .
\end{aligned}
$$

From the first line of this equation, we have $V \simeq v A$, so that we obtain, from Eq. (33),

$$
\xi=\frac{c A}{V} \simeq \frac{c A}{v A}=\frac{1}{\varepsilon} .
$$

Therefore, in the quasistatic limit $\varepsilon<<1$, this equation gives $\xi>>1$, so that the first line is compatible with the magnetic limit. Accordingly, this is incompatible with the electric limit, for which $\xi<<1$, so that the term $\mathbf{v} \cdot \mathbf{A}$ must be dropped from the first line of Eq. (37) in the electric limit.

A similar argument, applied to the second line of Eq. (37), implies $A \simeq \frac{v V}{c^{2}}$, so that

$$
\xi=\frac{c A}{V} \simeq \frac{c v V}{c^{2} V}=\frac{v}{c}=\varepsilon .
$$

Unlike the previous case, the quasistatic limit leads to $\xi<<1$, which is compatible with the electric limit only, Eq. (12). This implies that, in the magnetic limit, the term $\frac{\mathbf{v}}{c^{2}} V$ must be dropped from the second line of Eq. (37), as it is the case in Eq. (10).

If we use an entirely similar analysis for the Lorentz transformation of charge and current densities, obtained from Eq. (3) with $u^{0}=\rho$ and $\mathbf{u}=\mathbf{j} / c$ :

$$
\begin{aligned}
& \rho^{\prime}=\gamma\left(\rho-\frac{1}{c^{2}} \mathbf{v} \cdot \mathbf{j}\right), \\
& \mathbf{j}^{\prime}=\mathbf{j}-\gamma \mathbf{v} \rho+(\gamma-1) \frac{\mathbf{v}}{\mathbf{v}^{2}} \mathbf{v} \cdot \mathbf{j},
\end{aligned}
$$


we retrieve the Galilean transformations Eqs. (14) and (16) for the magnetic and electric limits, respectively.

Let us conclude by briefly discussing the continuity equation:

$$
\nabla \cdot \mathbf{j}+\partial_{t} \rho=0
$$

If we compare the two terms as we have done for the Lorentz gauge condition in Eq. (34), we find

$$
\frac{|\nabla \cdot \mathbf{j}|}{\partial_{t} \rho} \simeq \frac{c T}{L} \frac{j}{c \rho} \simeq \frac{\xi}{\varepsilon}
$$

If $\xi<<1$, like $\varepsilon$ in the quasistatic regime $\varepsilon<<1$, then we obtain the electric limit and we retrieve Eq. (17). On the other hand, in the magnetic case, $\xi>>1$, so that we drop $\partial_{t} \rho$ and obtain thereby Eq. (15).

\subsection{Reduction from $(4,1)$ Minkowski space-time}

Hereafter we review briefly a different approach to the Galilean gauge fields [16]. It involves a formulation of Galilean invariance based on a reduction from a fivedimensional Minkowski manifold to the Newtonian space-time [17]-[19]. The extended space is such that a Galilean boost with relative velocity $\mathbf{v}=\left(v_{1}, v_{2}, v_{3}\right)$ acts on a Galilei-vector $(\mathbf{x}, t, s)$ as

$$
\begin{aligned}
& \mathbf{x}^{\prime}=\mathbf{x}-\mathbf{v} t \\
& t^{\prime}=t \\
& s^{\prime}=s-\mathbf{v} \cdot \mathbf{x}+\frac{1}{2} \mathbf{v}^{2} t
\end{aligned}
$$

Since $\partial_{s}$ tranforms like the mass $m$ (see below for a justification), one can see the additional coordinate $s$ as being conjugate to the mass $m$ since both are invariant under Galilean transformations. $s$ may be seen also as the action per unit mass. More about classical and quantum physical interpretations of $s$ is in Refs. [16]-[19].

The scalar product,

$$
(A \mid B)=A^{\mu} B_{\mu} \equiv \mathbf{A} \cdot \mathbf{B}-A_{4} B_{5}-A_{5} B_{4}
$$

of two Galilei-vectors $A$ and $B$ is invariant under the transformation, Eq. (39). This suggests a method to base the tensor calculus on the metric

$$
g^{\mu \nu}=g_{\mu \nu}=\left(\begin{array}{ccccc}
1 & 0 & 0 & 0 & 0 \\
0 & 1 & 0 & 0 & 0 \\
0 & 0 & 1 & 0 & 0 \\
0 & 0 & 0 & 0 & -1 \\
0 & 0 & 0 & -1 & 0
\end{array}\right) .
$$

Hereafter we refer to this as the Galilean metric. 
The transformation in Eq. (39) can be written in matrix form for the components of any five-vector as

$$
x^{\prime \mu}=\Lambda_{\nu}^{\mu} x^{\nu},
$$

where $\mu$ denotes the row and $\nu$ the column (so that $\Lambda^{\mu}{ }_{\nu}$ is the $(\mu \nu)$-entry) or

$$
\left(\begin{array}{l}
x^{\prime 1} \\
x^{\prime 2} \\
x^{\prime 3} \\
x^{\prime 4} \\
x^{\prime 5}
\end{array}\right)=\left(\begin{array}{ccccc}
1 & 0 & 0 & -v_{1} & 0 \\
0 & 1 & 0 & -v_{2} & 0 \\
0 & 0 & 1 & -v_{3} & 0 \\
0 & 0 & 0 & 1 & 0 \\
-v_{1} & -v_{2} & -v_{3} & \frac{1}{2} \mathbf{v}^{2} & 1
\end{array}\right)\left(\begin{array}{c}
x^{1} \\
x^{2} \\
x^{3} \\
x^{4} \\
x^{5}
\end{array}\right)
$$

For a five-oneform, this transformation reads

$$
x_{\mu}^{\prime}=\Lambda_{\mu}^{\nu} x_{\nu}
$$

where $\mu$ now denotes the column and $\nu$ the row (that is $\Lambda_{\mu}{ }^{\nu}$ is the $(\nu \mu)$-entry), or

$$
\left(x_{1}^{\prime}, x_{2}^{\prime}, x_{3}^{\prime}, x_{4}^{\prime}, x_{5}^{\prime}\right)=\left(x_{1}, x_{2}, x_{3}, x_{4}, x_{5}\right)\left(\begin{array}{ccccc}
1 & 0 & 0 & v_{1} & 0 \\
0 & 1 & 0 & v_{2} & 0 \\
0 & 0 & 1 & v_{3} & 0 \\
0 & 0 & 0 & 1 & 0 \\
v_{1} & v_{2} & v_{3} & \frac{1}{2} \mathbf{v}^{2} & 1
\end{array}\right) .
$$

We write the embedding as

$$
(\mathbf{x}, t) \rightarrow x^{\mu}=(\mathbf{x}, t, s)
$$

as well as the following five-momentum:

$$
p_{\mu} \equiv-i \partial_{\mu}=\left(-i \nabla,-i \partial_{t},-i \partial_{s}\right)
$$

so that, with the usual identification $E=i \partial_{t}$, and with $m=i \partial_{s}$, we obtain

$$
\begin{aligned}
& p_{\mu}=(\mathbf{p},-E,-m), \\
& p^{\mu}=g^{\mu \nu} p_{\nu}=(\mathbf{p}, m, E) .
\end{aligned}
$$

Thereupon the mass does not enter as an external parameter, but as a remnant of the fifth component of the particle's momentum. Hereafter, the five-momentum operator will act on a massless field, so that

$$
\partial_{5} A=\partial_{s} A=0
$$

Now let us set up the five-dimensional quantities that allow us to retrieve the two Galilean limits of electromagnetism. They are given by defining two embeddings of the five-potential:

$$
A_{\mu}=\left(\mathbf{A}, A_{4}, A_{5}\right)
$$


Under the transformation in Eq. (39) its components transform, from Eq. (41), as

$$
\begin{aligned}
& \mathbf{A}^{\prime}=\mathbf{A}+\mathbf{v} A_{5}, \\
& A_{4^{\prime}}=A_{4}+\mathbf{v} \cdot \mathbf{A}+\frac{1}{2} \mathbf{v}^{2} A_{5}, \\
& A_{5^{\prime}}=A_{5} .
\end{aligned}
$$

Next we write the five-dimensional electromagnetic antisymmetric Faraday tensor:

$$
F_{\mu \nu} \equiv \partial_{\mu} A_{\nu}-\partial_{\nu} A_{\mu}=\left(\begin{array}{ccccc}
0 & b_{3} & -b_{2} & c_{1} & d_{1} \\
-b_{3} & 0 & b_{1} & c_{2} & d_{2} \\
b_{2} & -b_{1} & 0 & c_{3} & d_{3} \\
-c_{1} & -c_{2} & -c_{3} & 0 & a \\
-d_{1} & -d_{2} & -d_{3} & -a & 0
\end{array}\right) .
$$

Thus we have

$$
\begin{aligned}
& \mathbf{b}=\nabla \times \mathbf{A}, \\
& \mathbf{c}=\nabla A_{4}-\partial_{4} \mathbf{A}, \\
& \mathbf{d}=\nabla A_{5}-\partial_{5} \mathbf{A}, \\
& a=\partial_{4} A_{5}-\partial_{5} A_{4} .
\end{aligned}
$$

The five-current

$$
j_{\mu}=\left(\mathbf{j}, j_{4}, j_{5}\right),
$$

transforms under the transformation, Eq. (39), as

$$
\begin{aligned}
& \mathbf{j}^{\prime}=\mathbf{j}+\mathbf{v} j_{5}, \\
& j_{4^{\prime}}=j_{4}+\mathbf{v} \cdot \mathbf{j}+\frac{1}{2} \mathbf{v}^{2} j_{5}, \\
& j_{5^{\prime}}=j_{5} .
\end{aligned}
$$

The continuity equation takes the form

$$
\partial^{\mu} j_{\mu}=\nabla \cdot \mathbf{j}-\partial_{4} j_{5}-\partial_{5} j_{4}=0 .
$$

The five-dimensional Lorenz-like condition takes a similar form:

$$
\partial^{\mu} A_{\mu}=\nabla \cdot \mathbf{A}-\partial_{4} A_{5}-\partial_{5} A_{4}=0 .
$$

In the presence of sources, the Maxwell equations are

$$
\partial_{\mu} F_{\alpha \beta}+\partial_{\alpha} F_{\beta \mu}+\partial_{\beta} F_{\mu \alpha}=0,
$$

and

$$
\partial_{\nu} F^{\mu \nu}=j^{\mu},
$$

so that in terms of the components of $F$ defined in Eq. (43), we find, from Eq. (48):

$$
\begin{aligned}
& \nabla \cdot \mathbf{b}=0, \\
& \nabla \times \mathbf{c}+\partial_{4} \mathbf{b}=\mathbf{0}, \\
& \nabla \times \mathbf{d}+\partial_{5} \mathbf{b}=\mathbf{0}, \\
& \nabla a-\partial_{4} \mathbf{d}+\partial_{5} \mathbf{c}=\mathbf{0},
\end{aligned}
$$


whereas Eq. (49) reduces to

$$
\begin{aligned}
& \nabla \times \mathbf{b}-\partial_{5} \mathbf{c}-\partial_{4} \mathbf{d}=\mathbf{j}, \\
& \nabla \cdot \mathbf{c}-\partial_{4} a=-j_{4}, \\
& \nabla \cdot \mathbf{d}+\partial_{5} a=-j_{5} .
\end{aligned}
$$

From $F_{\mu^{\prime} \nu^{\prime}}=\Lambda_{\mu^{\prime}}^{\alpha} \Lambda_{\nu^{\prime}}^{\beta} F_{\alpha \beta}$ the entries of $F$ in Eq. (41) transform as

$$
\begin{aligned}
& a^{\prime}=a+\mathbf{v} \cdot \mathbf{d}, \\
& \mathbf{b}^{\prime}=\mathbf{b}-\mathbf{v} \times \mathbf{d} \\
& \mathbf{c}^{\prime}=\mathbf{c}+\mathbf{v} \times \mathbf{b}+\frac{1}{2} \mathbf{v}^{2} \mathbf{d}-a \mathbf{v}-\mathbf{v}(\mathbf{v} \cdot \mathbf{d}) \\
& \mathbf{d}^{\prime}=\mathbf{d}
\end{aligned}
$$

Let us now see how the electric and magnetic limits are contained within the previous formulas.

\subsubsection{Electric limit}

As mentioned previously, the electric limit is characterized by four-potential and fourcurrent vectors which are timelike, that is, their time component is much larger than the length of their spatial components. In the reduction approach, it corresponds to defining the embedding of the potentials and currents as

$$
\left(\mathbf{A}_{e}, V_{e}\right) \hookrightarrow A_{e}=\left(\mathbf{A}_{e}, 0,-\mu_{0} \epsilon_{0} V_{e}\right),
$$

and

$$
\left(\mathbf{j}_{e}, \rho_{e}\right) \hookrightarrow j_{e}=\left(\mu_{0} \mathbf{j}_{e}, 0,-\mu_{0} \rho_{e}\right)
$$

respectively.

From Eqs. (42) and (53) we retrieve Eq. (12). Similarly we obtain Eq. (16) from Eqs. (45) and (54). As for the continuity equation, Eq. (46), it becomes Eq. (17). From the first line of Eq. (44), we come to the natural definition:

$$
\mathbf{B}_{e} \equiv \mathbf{b}=\nabla \times \mathbf{A}_{e} .
$$

The electric field is defined as the component $\mathbf{d}$, so that from the third line of Eq. (44) we have $\mathbf{E}_{e} \equiv \frac{1}{\mu_{0} \epsilon_{0}} \mathbf{d}=-\nabla V_{e}$, as in Eq. (13). From Eq. (44) we note that

$\mathbf{c}=-\partial_{t} \mathbf{A}_{e}$ and $a=-\frac{1}{\mu_{0} \epsilon_{0}} \partial_{t} V_{e}$. Then Eq. (52) leads to Eq. (8). The corresponding Maxwell equations, Eq. (19), are obtained from Eqs. (50) and (51).

Note that the second line of Eq. (51) provides a condition similar to Lorenz gauge fixing:

$$
\nabla \cdot \mathbf{A}_{e}=-\mu_{0} \epsilon_{0} \partial_{t} V_{e}
$$

This expression may be obtained also by substituting Eq. (53) into Eq. (47). 


\subsubsection{Magnetic limit}

This non-relativistic limit is characterized by spacelike four-potential and four-current vectors; their time component is small compared to the length of their spatial components. Hereafter we show that it corresponds to defining the embedding of the potentials and currents as

$$
\left(\mathbf{A}_{m}, V_{m}\right) \hookrightarrow A_{m}=\left(\mathbf{A}_{m},-V_{m}, 0\right),
$$

and

$$
\left(\mathbf{j}_{m}, \rho_{m}\right) \hookrightarrow j_{m}=\left(\mu_{0} \mathbf{j}_{m},-\frac{1}{\epsilon_{0}} \rho_{m}, 0\right)
$$

respectively.

From Eqs. (42) and (55) we retrieve Eq. (10). Similarly Eqs. (45) and (56) lead to Eq. (14), and the continuity equation (46) gives Eq. (15), which shows that the current $\mathbf{j}_{m}$ cannot be related to a convective transport of charge! As above, we define the magnetic field as $\mathbf{B}_{m} \equiv \mathbf{b}=\nabla \times \mathbf{A}_{m}$, and the electric field is now defined as the component $\mathbf{c}$, so that from the second line of Eq. (44) we obtain $\mathbf{E}_{m} \equiv \mathbf{c}=-\nabla V_{m}-\partial_{t} \mathbf{A}_{m}$, as in Eq. (11). Then Eq. (52) leads to Eq. (7). The Maxwell equations (20) are obtained from Eqs. (50) and (51). Finally, note that by replacing Eq. (55) into Eq. (47) we obtain Coulomb's gauge condition:

$$
\nabla \cdot \mathbf{A}_{m}=0 .
$$

\section{Discussion and examples}

\subsection{Gauge conditions and Galilean electromagnetism}

In this section, we describe the two Galilean limits using the Riemann-Lorentz approach, that is, in terms of the scalar and vector potentials. The definition $\mathbf{E}=$ $-\partial_{t} \mathbf{A}-\nabla V$ of Eq. (11) takes different forms in the Galilean limit, depending on the order of magnitude of each term. This is because the Galilean transformations for the potentials depend on whether we take the electric or magnetic limit. Let us evaluate the order of magnitude of the ratio between its two terms:

$$
\frac{\partial_{t} \mathbf{A}}{\nabla V} \simeq \frac{\frac{A}{T}}{\frac{V}{L}} \simeq \frac{L}{c T} \frac{c A}{V} \simeq \varepsilon \xi .
$$

In the magnetic limit, for which $\xi>>1$, this equation leads to $\mathbf{E}_{m}=-\nabla V_{m}-$ $\partial_{t} \mathbf{A}_{m}$ as in Eq. (11). By computing the curl, we find $\partial_{t} \mathbf{B}_{m}=-\nabla \times \mathbf{E}_{m}$, as in the first line of Eq. (20). Likewise, in the electric limit, for which $\xi<<1$, we can neglect $\partial_{t} \mathbf{A}$ so that we find $\mathbf{E}_{e} \simeq-\nabla V_{e}$, as stated in Eq. (13). The curl of this expression leads to $\nabla \times \mathbf{E}_{e} \simeq \mathbf{0}$, as in the first line of Eq. (19). 
Let us illustrate how the choice of the gauge condition allows one to retrieve the two sets of "Galilean Maxwell equations" in terms of the fields, as stated by LBLL [1]. In the magnetic limit, the condition $\xi>>1$ leads to Eq. (36), as mentioned earlier. From the definition of $\mathbf{B}_{m}$ and using the identity

$$
\nabla \times(\nabla \times \mathbf{A})=\nabla(\nabla \cdot \mathbf{A})-\nabla^{2} \mathbf{A},
$$

we obtain

$$
\nabla \times \mathbf{B}_{m}=\nabla \times\left(\nabla \times \mathbf{A}_{m}\right)=\nabla(\overbrace{\nabla \cdot \mathbf{A}_{m}}^{0})-\nabla^{2} \mathbf{A}_{m}=\mu_{0} \mathbf{j}_{m},
$$

where the last term follows from Eq. (32). This is the third line of Eq. (20), where the displacement current term is missing. With $\mathbf{E}_{m}$ defined as in Eq. (11), its divergence gives

$$
\nabla \cdot \mathbf{E}_{m}=\nabla \cdot\left(-\partial_{t} \mathbf{A}_{m}-\nabla V_{m}\right)=-\partial_{t}(\overbrace{\nabla \cdot \mathbf{A}_{m}}^{0})-\nabla^{2} V_{m}=\frac{\rho_{m}}{\epsilon_{0}},
$$

where we have utilized again Eq. (36). This is the second inhomogeneous equation, last line of Eq. (20).

In the electric limit, $\xi<<1$ and Eq. (34) lead to the Lorenz condition, Eq. (35). Proceeding as in the magnetic limit, we first calculate the curl of $\mathbf{B}_{e}$, which gives us

$$
\nabla \times \mathbf{B}_{e}=\nabla \times\left(\nabla \times \mathbf{A}_{e}\right)=\nabla(\overbrace{\nabla \cdot \mathbf{A}_{e}}^{-\left(\partial_{t} V_{e}\right) / c^{2}})-\nabla^{2} \mathbf{A}_{e}=\frac{1}{c^{2}} \partial_{t} \mathbf{E}_{e}+\mu_{0} \mathbf{j}_{e} .
$$

We have utilized Eq. (13) to define $\mathbf{E}_{e}$. This is line three of Eq. (19). Finally, by calculating the divergence of $\mathbf{E}_{e}$, we find

$$
\nabla \cdot \mathbf{E}_{e}=\nabla \cdot\left(-\nabla V_{e}\right)=-\nabla^{2} V_{e}=\frac{\rho_{e}}{\epsilon_{0}},
$$

where we have used Eqs. (13) and (32).

Therefore, let us point out forcefully that the choice of a gauge condition is dictated by the relativistic versus Galilean nature of the problem. The Lorenz gauge condition must be chosen in the relativistic context as well as in the electric Galilean limit. The Coulomb gauge condition can be chosen only in the magnetic limit. For example, this implies that quantization in the Coulomb gauge of light waves is prohibited because of Galilean covariance and must be re-examined. We refer to a discussion of the physical meaning that one can ascribe to the various gauge conditions [14].

From the historical point of view, Galilean electromagnetism has shed a new light on the pre-relativity era. Indeed, a careful reading of James Clerk Maxwell's famous Treatise on Electricity and Magnetism reveals that he has employed the electric limit when dealing with dielectrics in his first volume [20]. On the other hand, in his second volume, he used the magnetic limit when dealing with ohmic conductors, except 
toward the end, where he introduced into the magnetic limit equations the so-called displacement current 'by hand' in order to demonstrate that light is a transverse electromagnetic wave [20]. But, as we have seen in the particular case of the electric limit (and it is also valid in relativity), the displacement current follows from choosing the Lorenz gauge whereas Maxwell (wrongfully!) kept the Coulomb gauge within the relativistic context for the fields. This difficulty enticed Hertz and Heaviside to abandon potentials and to cast Maxwell's equations in terms of fields rather than potentials. Albert Einstein read Hertz' papers on the topic and subsequently employed Maxwell equations in terms of the fields (the Heaviside-Hertz formulation) whereas Henri Poincaré employed Maxwell equations in terms of the potentials (the RiemannLorenz formulation) by adopting the Lorenz condition in a relativistic context [2].

\subsection{Magnetoquasistatics (MHD) and electroquasitatics (EHD)}

Experts working on magnetohydrodynamics (MHD) and experts of electrohydrodynamics (EHD) might be surprised to realize that they actually work with different sets of approximations of the Maxwell equations where retardation (and, therefore, waves) has been neglected. Indeed, the displacement current is negligible in MHD (as in Eq. (20)), whereas in EHD the electric field has a vanishing curl (Eq. (19). Hence, effects that are important in MHD becomes marginal in EHD, and vice versa.

Melcher has greatly clarified these facts by disjoining the electroquasistationary approximation used in EHD and the magnetoquasistationary approximation used in MHD [21] Section 3.2 of Melcher and Haus [21] shows that the underlying equations are precisely the same as the Galilean limits, Eqs. (19) and (20). His main argument relies on the comparison between three characteristics time scales ( $\sigma$ is the electric conductivity): (1) the magnetic diffusion time $\tau_{m}=\mu_{0} \sigma L^{2}$, (2) the charge relaxation time $\tau_{e}=\epsilon_{0} / \sigma$ and (3) the wave transit time $\tau_{e m}=L / c=\sqrt{\tau_{e} \times \tau_{m}}$, which is the square root of the product of the two former time scales. By definition, the transit time is always between the magnetic and electric times. For example, in the magnetic limit, the charge relaxation time scale is very small and the magnetic field does have enough time to diffuse inside the Ohmic carrier. It is straightforward to see that the electroquasistatic of Melcher corresponds to the electric limit whereas the magnetic limit is just the magnetoquasistatic. Hence, a large amount of our technology is based on Galilean electromagnetism as soon as waves are neglected.

\subsection{The Faraday tensor and its dual}

It is well known that in special relativity, the Faraday tensor, defined in Eq. (43):

$$
F_{\mu \nu} \equiv \partial_{\mu} A_{\nu}-\partial_{\nu} A_{\mu},
$$

(in this section, $\mu, \nu=0,1,2,3$ ) and its dual:

$$
{ }^{*} F_{\mu \nu}=\frac{1}{2} \epsilon^{\mu \nu \rho \sigma} F_{\rho \sigma}
$$


do have the same physical meaning. This is not the case within Galilean electromagnetism. Indeed, as pointed out by Earman and subsequently discussed by Rynasiewicz, the Galilean tranformations of the Faraday tensor and its dual lead to the electric or the magnetic limit, respectively [22]. The effect of this duality operation amounts to exchanging $\mathbf{E}$ and $\mathbf{B}$ as follows:

$$
\mathbf{E} \rightarrow c \mathbf{B} \text { and } \mathbf{B} \rightarrow-\mathbf{E} / c .
$$

One recovers very easily the magnetic and electric limits, Eqs. (7) and (8), by applying the duality transformations directly to the electric transformations of the field in order to get the magnetic transformations, and vice versa.

It is also noted in Ref. [22] that the field transformations of the magnetic limit are obtained when $\mathbf{E}$ and $\mathbf{B}$ are expressed in terms of 'covariant', or $\left(\begin{array}{l}0 \\ 2\end{array}\right)$, tensor $F_{\mu \nu}$, whereas the electric limit is obtained when the fields transformations are calculated by using the 'contravariant', or $\left(\begin{array}{l}2 \\ 0\end{array}\right)$, tensor $F^{\mu \nu}$. Let us illustrate it briefly, with

$$
A^{\mu}=\left(\frac{V}{c}, \mathbf{A}\right), \quad A_{\mu}=\left(\frac{V}{c},-\mathbf{A}\right)
$$

as well as

$$
\partial^{\mu}=\left(\frac{1}{c} \partial_{t},-\nabla\right), \quad \partial_{\mu}=\left(\frac{1}{c} \partial_{t}, \nabla\right)
$$

The magnetic limit rests on the relation

$$
F_{\mu \nu}^{\prime}=\Lambda_{\mu}^{\rho} \Lambda_{\nu}^{\sigma} F_{\rho \sigma}
$$

where the Galilean transformation matrix $\Lambda_{\mu}{ }^{\nu}$ is defined by the four-gradient transformation, $\partial_{\mu}^{\prime}=\Lambda_{\mu}^{\nu} \partial_{\nu}$, so that

$$
\Lambda_{\mu}{ }^{\nu}=\left(\begin{array}{cccc}
1 & \frac{v_{x}}{c} & \frac{v_{y}}{c} & \frac{v_{z}}{c} \\
0 & 1 & 0 & 0 \\
0 & 0 & 1 & 0 \\
0 & 0 & 0 & 1
\end{array}\right)
$$

The index $\mu$ denotes the line of each entry. We find, for example,

$$
\begin{aligned}
\frac{E_{x}^{\prime}}{c}=F_{01}^{\prime} & =\Lambda_{0}^{\mu} \Lambda_{1}{ }^{\nu} F_{\mu \nu} \\
& =\Lambda_{0}^{0} F_{01}+\Lambda_{0}^{2} F_{21}+\Lambda_{0}^{3} F_{31} \\
& =\frac{1}{c}\left(E_{x}+v_{y} B_{z}-v_{z} B_{y}\right)
\end{aligned}
$$

and

$$
-B_{z}^{\prime}=F_{12}^{\prime}=\Lambda_{1}^{\mu} \Lambda_{2}{ }^{\nu} F_{\mu \nu}=-B_{z}
$$

which is Eq. (7). 
The electric limit transformations follows from

$$
F^{\prime \mu \nu}=\Lambda_{\rho}^{\mu} \Lambda_{\sigma}^{\nu} F^{\rho \sigma}
$$

The transformation matrix $\Lambda_{\nu}^{\mu}$ is now defined by the coordinate transformation, $x^{\mu}=\Lambda_{\nu}^{\mu} x^{\nu}$, with $x^{\mu}=(c t, x, y, z)$, so that

$$
\Lambda_{\nu}^{\mu}=\left(\begin{array}{cccc}
1 & 0 & 0 & 0 \\
-\frac{v_{x}}{c} & 1 & 0 & 0 \\
-\frac{v_{y}}{c} & 0 & 1 & 0 \\
-\frac{v_{z}}{c} & 0 & 0 & 1
\end{array}\right)
$$

Again, the first index $\mu$ denotes the matrix line. For instance, we compute

$$
-\frac{E_{x}^{\prime}}{c}=F^{\prime 01}=\Lambda_{0}^{0} \Lambda^{1}{ }_{\nu} F^{0 \nu}=-\frac{E_{x}}{c},
$$

and

$$
\begin{aligned}
B_{z}^{\prime}=-F^{\prime 2} & =-\Lambda^{1}{ }_{\mu} \Lambda^{2}{ }_{\nu} F^{\mu \nu} \\
& =-\Lambda^{1}{ }_{0} F^{02}-\Lambda^{2}{ }_{0} F^{10}-F^{12} \\
& =B_{z}-\frac{1}{c^{2}}\left(v_{x} E_{y}-v_{y} E_{x}\right)
\end{aligned}
$$

which is Eq. (8).

\subsection{Quantum mechanics}

In 1990, Dyson published a demonstration of Maxwell equations due to Richard Feynman [23]. This demonstration, which relied on Lagrangians and quantum mechanics, dated back to the forties and had remained hitherto unpublished. However, the proof was believed to be incomplete because Feynman had discussed only the homogeneous Maxwell equations, given by the first two lines in Eq. (18):

$$
\nabla \times \mathbf{E}=-\partial_{t} \mathbf{B}, \quad \nabla \cdot \mathbf{B}=0 .
$$

In 1999, Brown and Holland revisited this demonstration and they have noted the Schrödinger equation admitted external potentials only if they were compatible with the magnetic limit of LBLL and, therefore, with the Coulomb gauge condition [24]. It is clear from Eq. (20) that the homogeneous Maxwell equations given above are valid only within the magnetic limit, because the electric field has zero curl in the electric limit, Eq. (19). This is a consequence of the Galilean magnetic limit of the fourpotential which does enters into Schrödinger equation. Let us recall the statement of Brown and Holland more precisely. The Schrödinger equation with external fields $V(\mathbf{x}, t)$ and $\mathbf{A}(\mathbf{x}, t)$ :

$$
\mathrm{i} \hbar \partial_{t} \Psi(\mathbf{x}, t)=\frac{1}{2 m}(-\mathrm{i} \hbar \nabla-\mathbf{A}(\mathbf{x}, t))^{2} \Psi(\mathbf{x}, t)+V(\mathbf{x}, t) \Psi(\mathbf{x}, t),
$$


is covariant under Galilean transformation, Eq. (6), with $\Psi(\mathbf{x}, t) \rightarrow \Psi^{\prime}\left(\mathbf{x}^{\prime}, t^{\prime}\right), V(\mathbf{x}, t) \rightarrow$ $V^{\prime}\left(\mathbf{x}^{\prime}, t^{\prime}\right)$, and $\mathbf{A}(\mathbf{x}, t) \rightarrow \mathbf{A}^{\prime}\left(\mathbf{x}^{\prime}, t^{\prime}\right)$, if

$$
\begin{aligned}
\Psi^{\prime}\left(\mathbf{x}^{\prime}, t^{\prime}\right) & =\text { const } \exp \left[(\mathrm{i} / \hbar)\left(-m \mathbf{v} \cdot \mathbf{x}+\frac{1}{2} m \mathbf{v}^{2} t+\phi(\mathbf{x}, t)\right)\right] \Psi(\mathbf{x}, t) \\
V^{\prime}\left(\mathbf{x}^{\prime}, t^{\prime}\right) & =V(\mathbf{x}, t)-\partial_{t} \phi(\mathbf{x}, t)-\mathbf{v} \cdot(\mathbf{A}(\mathbf{x}, t)+\nabla \phi(\mathbf{x}, t)) \\
\mathbf{A}^{\prime}\left(\mathbf{x}^{\prime}, t^{\prime}\right) & =\mathbf{A}(\mathbf{x}, t)+\nabla \phi(\mathbf{x}, t)
\end{aligned}
$$

where $\phi(\mathbf{x}, t)$ is some scalar function. Note that the factor in front of $\Psi(\mathbf{x}, t)$ transforms like the parameter $s$ in Eq. (39). If we choose $\phi(\mathbf{x}, t)=0$, then the equations above reduce to Eq. (10), which represents the Galilean transformations of the potentials in the magnetic limit.

This point agrees with later results by Vaidya and Farina [25]. In a subsequent study, Holland and Brown have shown that Maxwell equations admit an electric limit only if the source is a Dirac current [26]. In addition, they have proved that the Dirac equation admits both Galilean limits, just like Maxwell equations, corroborating thereby earlier results by Lévy-Leblond [27]. What Feynmann did not (actually, could not) realize is that he had derived only the part of Maxwell equations compatible with both Galilean relativity and quantum mechanics, that is, the magnetic limit and, hence, the homogeneous equations.

\subsection{Superconductivity}

Superconductivity also enters into the realm of the magnetic limit, because it selects the Coulomb gauge condition as a necessary consequence of Galilean covariance. Indeed, the well-known London equation states that the current density is proportional to the vector potential (the star denotes Cooper pairs): $\mathbf{p}=m^{*} \mathbf{v}+q^{*} \mathbf{A}=\mathbf{0}$. As a matter of fact, there is a perfect transfert of electromagnetic momentum to kinetic momentum. Hence, contrary to what is usually stated, gauge invariance is not broken by superconductivity since the Coulomb gauge condition is implied. Moreover, the Meissner effect can be explained by starting with Ampère's equation written as $\nabla \times \mathbf{B}=\mu_{0} \mathbf{j}$, that is, without displacement current term as in the magnetic case, third line of Eq. (20). Hence, this expression (or more directly $\nabla^{2} \mathbf{A} \simeq-\mu_{0} \mathbf{j}$ in the Riemann-Lorenz formulation) together with $\nabla \cdot \mathbf{A}=0$ and London equation, leads to solutions (in one dimension $x$ ) of the type $A \simeq \exp -$ const $x$ so that the vector potential (hence the magnetic field) only penetrates the superconductor to a depth $1 /$ const.

Superconductivity cannot be associated to a symmetry breaking of gauge invariance but is magnetic Galilean covariant. This unusual statement has been recently advocated by Martin Greiter by a different approach [28]. As a matter of fact, it is the global $\mathrm{U}(1)$ phase rotation symmetry that is spontaneously violated. A striking consequence is that the Higgs mechanism for providing mass to particles becomes doubtful, since it was believed to be analogous to the assumed symmetry breaking of gauge invariance in superconductivity. 


\subsection{Electrodynamics of continuous media at low velocities}

One often finds in textbooks that a dielectric in motion is characterized by the presence of a motional polarization given by the following formula [29]:

$$
\mathbf{P}^{\prime}=\epsilon_{0} \chi(\mathbf{E}+\mathbf{v} \times \mathbf{B}) .
$$

where $\chi$ is the dielectric susceptibility. For example, H.A. Lorentz utilized it in order to derive the Fresnel-Fizeau formula at first order (see p. 174-176 of Ref. [29] and Ref. [30]). We will see that this formula can be misleading.

Moreover, in 1904, Lorentz claimed that a moving magnet could become electrically polarized [31]. In 1908, Einstein and Laub noted that Minkowski transformations for the fields and the excitations [32] predict that a moving magnetic dipole develops an electric dipole moment [33]. It would be interesting to reexamine these predictions in the light of Galilean electromagnetism within continuous media. Indeed, if one starts from the Minkowski transformations relating the polarization and the magnetization [32], one would expect two Galilean limits: one with $\mathbf{M}^{\prime}=\mathbf{M}$ and $\mathbf{P}^{\prime}=\mathbf{P}-\mathbf{v} \times \mathbf{M} / c^{2}$ and the other with $\mathbf{M}^{\prime}=\mathbf{M}+\mathbf{v} \times \mathbf{P}$ and $\mathbf{P}^{\prime}=\mathbf{P}$ (see Chapter 9 of Ref. [29]).

We can derive from the Riemann-Lorenz formulation Maxwell's equations within continuous media following O'Rahilly [12]. More directly, one can infer the form of Maxwell equations in continuous media by mimicking the vacuum case by either supressing the displacement current or the Faraday term.

Maxwell equations in continuous media are covariant under the Poincaré-Lorentz transformations [32]:

$$
\begin{aligned}
& \nabla \cdot \mathbf{B}=0, \\
& \partial_{t} \mathbf{B}=-\nabla \times \mathbf{E}, \\
& \nabla \cdot \mathbf{D}=\rho, \\
& \nabla \times \mathbf{H}=\mathbf{j}+\partial_{t} \mathbf{D} .
\end{aligned}
$$

In continuous media, the constitutive laws relate the excitation $\mathbf{D}$, the field $\mathbf{E}$ and the polarization $\mathbf{M}$ :

$$
\begin{aligned}
& \mathbf{D}=\epsilon_{0} \mathbf{E}+\mathbf{P}, \\
& \mathbf{B}=\mu_{0} \mathbf{H}+\mathbf{M} .
\end{aligned}
$$

These relations are valid in both Galilean and Einsteinian relativity [32]. Let us now turn to the electromagnetic laws when we take into account the motion of a medium at low velocity [21].

First, we recall the Galilean transformations for the differentiation operators:

$$
\begin{aligned}
\nabla^{\prime} \times(\cdots) & =\nabla \times(\cdots) \\
\nabla^{\prime} \cdot(\cdots) & =\nabla \cdot(\cdots) \\
\nabla^{\prime}(\cdots) & =\nabla(\cdots)
\end{aligned}
$$




$$
\begin{gathered}
\frac{\partial \mathbf{a}^{\prime}}{\partial t^{\prime}}=\frac{\partial \mathbf{a}^{\prime}}{\partial t}+(\mathbf{v} \cdot \nabla) \mathbf{a}^{\prime} \\
\nabla \times(\mathbf{a} \times \mathbf{b})=(\mathbf{b} \cdot \nabla) \mathbf{a}-(\mathbf{a} \cdot \nabla) \mathbf{b}+\mathbf{a}(\nabla \cdot \mathbf{b})-\mathbf{b}(\nabla \cdot \mathbf{a})
\end{gathered}
$$

If $\mathbf{a}=\mathbf{v}$ and $\mathbf{b}=\mathbf{A}^{\prime}$, then:

$$
\frac{\partial \mathbf{A}^{\prime}}{\partial t^{\prime}}=\frac{\partial \mathbf{A}^{\prime}}{\partial t}+\mathbf{v}\left(\nabla \cdot \mathbf{A}^{\prime}\right)-\nabla \times\left(\mathbf{v} \times \mathbf{A}^{\prime}\right)
$$

Let us apply these transformations to the two Galilean limits of Maxwell equations expressed for a continuous medium. We write first Maxwell equations in a frame of reference $R^{\prime}$ moving at a relative velocity $\mathbf{v}$ with respect to another frame $R$ :

$$
\begin{gathered}
\text { Magnetic Limit } \\
\hline \nabla^{\prime} \times \mathbf{H}^{\prime}=\mathbf{j}^{\prime} \\
\nabla^{\prime} \cdot \mathbf{B}^{\prime}=0 \\
\nabla^{\prime} \cdot \mathbf{j}^{\prime}=0 \\
\partial_{t^{\prime}} \mathbf{B}^{\prime}=-\nabla^{\prime} \times \mathbf{E}^{\prime}
\end{gathered}
$$

$$
\begin{gathered}
\frac{\text { Electric Limit }}{\nabla^{\prime} \times \mathbf{E}^{\prime}=\mathbf{0}} \\
\nabla^{\prime} \cdot \mathbf{D}^{\prime}=\rho^{\prime} \\
\nabla^{\prime} \cdot \mathbf{j}^{\prime}+\frac{\partial \rho^{\prime}}{\partial \mathrm{t}^{\prime}}=0 \\
\nabla^{\prime} \times \mathbf{H}^{\prime}=\mathbf{j}^{\prime}+\frac{\partial \mathbf{D}^{\prime}}{\partial t^{\prime}}
\end{gathered}
$$

We apply the spatial and temporal Galilean derivatives, so that in R', we find:

$$
\begin{gathered}
\frac{\text { Magnetic Limit }}{\nabla \times \mathbf{H}^{\prime}=\mathbf{j}^{\prime}} \\
\nabla \cdot \mathbf{B}^{\prime}=0 \\
\nabla \cdot \mathbf{j}^{\prime}=0 \\
\partial_{t} \mathbf{B}^{\prime}=-\nabla \times\left(\mathbf{E}^{\prime}-\mathbf{v} \times \mathbf{B}^{\prime}\right)
\end{gathered}
$$$$
\begin{gathered}
\frac{\text { Electric Limit }}{\nabla \times \mathbf{E}^{\prime}=\mathbf{0}} \\
\nabla \cdot \mathbf{D}^{\prime}=\rho^{\prime} \\
\nabla \cdot\left(\mathbf{j}^{\prime}+\rho^{\prime} \mathbf{v}\right)+\frac{\partial \rho^{\prime}}{\partial \mathrm{t}}=0 \\
\nabla \times\left(\mathbf{H}^{\prime}+\mathbf{v} \times \mathbf{D}^{\prime}\right)=\mathbf{j}^{\prime}+\rho \mathbf{v}+\frac{\partial \mathbf{D}^{\prime}}{\partial t}
\end{gathered}
$$

Hence, we deduce the fields transformations:

$$
\begin{gathered}
\frac{\text { Magnetic Limit }}{\mathbf{B}=\mathbf{B}^{\prime}} \\
\mathbf{j}=\mathbf{j}^{\prime} \\
\mathbf{H}=\mathbf{H}^{\prime} \\
\mathbf{E}=\mathbf{E}^{\prime}-\mathbf{v} \times \mathbf{B}^{\prime} \\
\mathbf{M}=\mathbf{M}^{\prime} \\
\mathbf{P}=\mathbf{P}^{\prime}+\mathbf{v} \times \mathbf{M}^{\prime} / c^{2}
\end{gathered}
$$

Electric Limit

$$
\begin{gathered}
\mathbf{E}=\mathbf{E}^{\prime} \\
\rho=\rho^{\prime} \\
\mathbf{j}=\mathbf{j}^{\prime}+\rho^{\prime} \mathbf{v} \\
\mathbf{H}=\mathbf{H}^{\prime}+\mathbf{v} \times \mathbf{D}^{\prime} \\
\mathbf{D}=\mathbf{D}^{\prime} \\
\mathbf{P}=\mathbf{P}^{\prime} \\
\mathbf{M}=\mathbf{M}^{\prime}-\mathbf{v} \times \mathbf{P}^{\prime}
\end{gathered}
$$


As suspected, the effects predicted by Lorentz and Einstein \& Laub in continuous media are of galilean origin and not relativistic !

In addition, we can derive easily the boundary conditions for moving media with $\mathbf{n}=\mathbf{n}_{1 \rightarrow 2}$ the unit vector between the two media denoted by 1 and 2

$$
\begin{gathered}
\frac{\text { Magnetic Limit }}{\times\left(\mathbf{H}_{2}-\mathbf{H}_{1}\right)=\mathbf{K}} \\
\mathbf{n} \cdot\left(\mathbf{B}_{2}-\mathbf{B}_{1}\right)=\mathbf{0} \\
\mathbf{n} \cdot\left(\mathbf{j}_{2}-\mathbf{j}_{1}\right)+\nabla_{\Sigma} \cdot \mathbf{K}=\mathbf{0} \\
\mathbf{n} \times\left(\mathbf{E}_{2}-\mathbf{E}_{1}\right)=v_{n}\left(\mathbf{B}_{2}-\mathbf{B}_{1}\right)
\end{gathered}
$$

$$
\begin{gathered}
\quad \stackrel{\text { Electric Limit }}{\mathbf{n} \times\left(\mathbf{E}_{2}-\mathbf{E}_{1}\right)=0} \\
\mathbf{n} \cdot\left(\mathbf{D}_{2}-\mathbf{D}_{1}\right)=\sigma \\
\mathbf{n} \cdot\left(\mathbf{j}_{2}-\mathbf{j}_{1}\right)+\nabla_{\Sigma} \cdot \mathbf{K}=v_{n}\left(\rho_{2}-\rho_{1}\right)-\partial_{t} \sigma \\
\mathbf{n} \times\left(\mathbf{H}_{2}-\mathbf{H}_{1}\right)=\mathbf{K}+v_{n} \mathbf{n} \times\left[\mathbf{n} \times\left(\mathbf{D}_{2}-\mathbf{D}_{1}\right)\right]
\end{gathered}
$$

$\mathbf{K}$ is the surface current, $\sigma$ the surface charge, $\Sigma$ the surface separating both media, and $v_{n}$ the projection of the relative velocity on the normal of $\Sigma$.

The formula, Eq. (57), used by Lorentz is not compatible with Galilean relativity. However, the electric field and the magnetic field which create the polarization in Fizeau experiment come from a light wave so that Lorentz was right to use this formula after all, even though there is no contradiction with the electric limit formula $\mathbf{P}^{\prime}=\mathbf{P}=\epsilon_{0} \chi \mathbf{E}^{\prime}=\epsilon_{0} \chi \mathbf{E}$.

\subsection{Electrodynamics of moving bodies at low velocities}

Galilean electromagnetism raises severe doubts concerning our current understanding of the electrodynamics of moving media. Indeed, several experiments, like the ones by Roentgen [34], Eichenwald [35], Wilson [36], Wilson and Wilson [37], Trouton and Noble [38], etc., were believed to corroborate special relativity. As we will demonstrate for the Trouton-Noble experiment, there is not always a need for special relativity because the typical relative velocity in these experiments is well below the speed of light. Then, the question is whether the above mentioned experiments be explained by either the electric limit, the magnetic limit or a combination of both.

\subsubsection{The Trouton-Noble experiment}

The Trouton-Noble's experiment is thought to be the electromagnetic analogue of the optical Michelson and Morley experiment [38]. It was designed in order to show whether one can observe a mechanical velocity of the ether if one considers that the luminiferous medium should be a medium whose parts can be followed mechanically. Like the null result of Michelson-Morley's optical experiment, the Trouton-Noble experiment was negative in the sense that one was not able to detect either an absolute motion with respect to the ether, or a partial entrainment, as believed by other various theories. 
In 1905, Albert Einstein suggested to consider the ether as superfluous since the experiments were not able to detect a mechanical motion of it. Others, like H. Poincaré and H.A. Lorentz, were reluctant to abandon the notion of ether as the bearer of the electromagnetic field, despite the fact that they have adopted the relativity principle. However, in 1920, Einstein recoursed to the ether as the bearer of the metric allowing the propagation of gravitational waves, at a conference in Leyden. Today, even though the ether is a banished word in modern science, one can use it as did the older Einstein in order to describe the vacuum with physical (though not mechanical) properties.

Before the advent of special relativity, Hertz, Wien, Abraham, Lorentz, Cohn and others have used the transformations given in Eq. (2), which is an incoherent mixture of the electric and magnetic Galilean limits [39]. As mentioned previously, these expressions do not even obey the group property of composition of transformations.

Trouton and Noble expected a positive effect when a charged capacitor is in motion with an angle $\theta$ between the plates and the velocity [38]. Indeed, the electric field in the frame of the capacitor generates a magnetic field in the ether frame ( $\mathbf{v}$ is the absolute velocity):

$$
\mathbf{B}^{\prime}=-\frac{1}{c^{2}} \mathbf{v} \times \mathbf{E}
$$

that is,

$$
B^{\prime}=\frac{1}{c^{2}} v E \sin \theta
$$

Hence, there is a localization of magnetic energy density inside a volume $d \mathcal{V}$ :

$$
\mathrm{d} W=\frac{1}{2} \frac{B^{\prime}}{\mu_{0}} \mathrm{~d} \mathcal{V}=\frac{1}{2} \frac{v^{2}}{c^{2}} \epsilon_{0} E^{2} \sin ^{2} \theta \mathrm{d} \mathcal{V} .
$$

With the volume of the capacitor given by $S l$, the total energy between the plates is

$$
W=\frac{1}{2} \frac{v^{2}}{c^{2}} \epsilon_{0} E^{2} \sin ^{2} \theta S l .
$$

If one denotes as $V=E / l$ the difference of potential between the plates, then the capacitor is submitted to the electrical torque

$$
\Gamma=-\frac{\mathrm{d} W}{\mathrm{~d} \theta}=-\frac{\epsilon_{0}}{2} \frac{V^{2} S}{l} \frac{v^{2}}{c^{2}} \sin (2 \theta)
$$

which is maximal for $\theta=45^{\circ}$ and zero for $\theta=90^{\circ}$. Hence, the plates must be perpendicular to the velocity. One does not observe such an effect in practice.

In order to understand what is wrong with the above demonstration, we first recall that the electric limit transforms as in Eq. (8). A straightforward application of these transformations is to note that the Biot-Savart law follows from the Coulomb law associated to the electric transformation of the magnetic field. Contrary to the 
transformations used by Lorentz and Trouton-Noble, they do respect the group additivity. Besides, these transformations are only compatible with the approximate set of Maxwell equations where the time derivative in the Faraday equation vanishes, as in Eq. (19). Hence, we can derive easily the approximate Poynting's theorem within the electric limit from this set:

$$
\partial_{t}\left(\frac{1}{2} \epsilon_{0} E^{2}\right)+\nabla \cdot\left(\frac{\mathbf{E} \times \mathbf{B}}{\mu_{0}}\right) \simeq-\mathbf{j} \cdot \mathbf{E} .
$$

As we can see, the energy density is of electrical origin only. Hence, no electric energy associated with the motional magnetic field can be taken into account within the electric limit since it is of order $v^{2} / c^{2}$ with respect to the static, or quasistatic, electric one. Thus, the Trouton-Noble experiment does not show any effect as soon as we are in the realm of the electric limit. We recall that the electric limit is such that the relative velocity is small compared to the velocity of light $c$, and the order of magnitude of the electric field is large compared to the product of $c$ by the magnetic field. Of course, for larger velocities, special relativity is needed and we must take into account additional mechanical torque due to lenght contraction as usual now in order to have a negative result (no torque).

The conclusion of Trouton and Noble is rather illuminating concerning the fact that they did consider the energy of the motional magnetic field to be the source of the negative result [38]:

"There is no doubt that the result is a purely negative one. As the energy of the magnetic field, if it exists (and from our present point of view we must suppose it does), must come from somewhere, we are driven to the conclusion that the electrostatic energy of a capacitor must dimininish by the amount $1 / 2 \epsilon_{0} E^{2} v^{2} / c^{2}$, when moving with a velocity $v$ at right angles to its electrostatic lines of force where $1 / 2 \epsilon_{0} E^{2}$ is the electrostatic energy."

Conversely, a solenoid/magnet in motion will not create a motionnal magnetic torque because the magnetic energy associated with the motional electric field is negligible compared to the magnetic energy of the static, or quasistatic, magnetic field.

\subsection{2 "Einstein asymmetry"}

In his famous article on the electrodynamics of moving media, Albert Einstein pointed out the importance of whether or not one should ascribe energy to the fields when dealing with motion [40]. We reproduced here the introduction of his paper:

"It is known that Maxwell's electrodynamics - as usually understood at the present time- when applied to moving bodies, leads to asymmetries which do not appear to be inherent in the phenomena. Take, for example, the reciprocal electrodynamic action 
of a magnet and a conductor. The observable phenomenon here depends only on the relative motion of the conductor and the magnet, whereas the customary view draws a sharp distinction between the two cases in which either one or the other of these bodies is in motion. For if the magnet is in motion and the conductor at rest, there arises in the neighbourhood of the magnet an electric field with a certain definite energy, producing a current at the places where parts of the conductor are situated. But if the magnet is stationary and the conductor in motion, then no electric field arises in the neighbourhood of the magnet. In the conductor, however, we find an electromotive force to which in itself there is no corresponding energy, but which gives rise -assuming equality of relative motion in the two cases discussed- to electric currents of the same path and intensity as those produced by the electric forces in the former case."

Like our discussion of the Trouton-Noble experiment, the magnetic Poynting's theorem can explain why one cannot ascribe an energy to the motional electric field in Einstein's thought experiment:

$$
\partial_{t}\left(\frac{B^{2}}{2 \mu_{0}}\right)+\nabla \cdot\left(\frac{\mathbf{E} \times \mathbf{B}}{\mu_{0}}\right) \simeq-\mathbf{j} \cdot \mathbf{E} .
$$

This is the magnetic analogue of Eq. (58).

By applying the Lorentz transformation that he had derived in the kinematical analysis of his article to the 'Maxwell' equations (in fact, he used the Heaviside-Hertz formulation, unlike Poincaré, who used the Riemann-Lorenz formulation in his relativity article), Einstein replaced Lorentz's explanation:

"1. If a unit electric point charge is in motion in an electromagnetic field, there acts upon it, in addition to the electric force, an electromotive force which, if we neglect the terms multiplied by the second and higher powers of $v / c$, is equal to the vector product of the velocity of the charge and the magnetic force, divided by the velocity of light. (Old manner of expression.)"

by the now famous special relativity explanation (valid for all velocities):

"2. If a unit electric point charge is in motion in an electromagnetic field, the force acting upon it is equal to the electric force which is present at the locality of the charge, and which we ascertain by transformation of the field to a system of coordinates at rest relatively to the electrical charge. (New manner of expression.)"

Then he concluded:

"The analogy holds with magnetomotive forces. We see that electromotive force plays in the developed theory merely the part of an auxiliary concept, which owes its 
introduction to the circumstance that electric and magnetic forces do not exist independently of the state of motion of the system of co-ordinates. Furthermore it is clear that the asymmetry mentioned in the introduction as arising when we consider the currents produced by the relative motion of a magnet and a conductor, now disappears. Moreover, questions as to the seat of electrodynamic electromotive forces (unipolar machines) now have no point."

However, we point out forcefully that the Galilean magnetic transformations of the electromagnetic field are sufficient to explain the magnet and conductor thought experiment of Albert Einstein as Lorentz covariance is not necessary [41]. It means that the second postulate (constancy of the velocity of light) used by Einstein was not necessary to explain the thought experiment. Only the relativity postulate and the magnetic Galilean transformations are necessary as the usual relative velocity of a real experiment is much more inferior to the velocity of light. Hence for low velocities regime, we proposed the following removal of Einstein's asymmetry:

"3. If a unit electric point charge is in motion in an electromagnetic field, the force acting upon it is equal to the electric force which is present at the locality of the charge, and which we ascertain by a Galilean magnetic transformation of the field to a system of co-ordinates at rest relatively to the electrical charge. (New manner of expression only valid for low velocities.)"

Einstein was right to replace Lorentz explanation as Lorentz thought that the cross product of the velocity and the magnetic field was not an electric field that's why the latter called it in particular the electromotive field. But Einstein did not see that the same cross product was an effective electric field due to a magnetic Galilean transformations. Wolfgang Pauli provided a resolution of the asymmetry in his textbook on electrodynamics but he only assumed that his calculations were a first order approximation of the relativistic demonstration without acknowledging the existence of a Galilean approximation as the magnetic limit [42]. Hence, Einstein's procedure to remove the asymmetry is completely valid despite the fact that special relativity is not necessary to remove it but only sufficient. Hence, ironically, the thought experiment who leaded Albert Einstein to special relativity could have been explained by Galilean relativity only with the use of the magnetic limit...

As pointed out a long time ago by Keswani and Kilminster [43], Maxwell did resolve Einstein's asymmetry within the formalism of the magnetic limit:

"In all phenomena relating to closed circuits and the current in them, it is indifferent whether the axes to which we refer the system be at rest or in motion".

Indeed, 
"The electromotive intensity is expressed by a formula of the same type, wheter the motions of the conductors be referred to fixed axes or to axes moving in space, the only differences between the formulae being that in the case of moving axes the electric potential $V$ must be changed into $V^{\prime}=V-\mathbf{v}$.A. In all cases in which a current is produced in a conducting circuit, the electromotive force is the line-integral $e=\int_{C} \mathbf{E}^{\prime} . d \mathbf{s}$ taken round the curve. The value of $V$ disappears from this integral, so that the introduction of -v.A has no influence on its value."

\section{Concluding remarks}

One century after the relativity revolution and more than thirty years after the forgotten work of Lévy-Leblond and Le Bellac, Galilean electromagnetism is becoming a field of actual research as we can explain much more simply scores of experiments involving the electrodynamics of moving media without the sophisticated formalism of special relativity.

Moreover, for slow velocities, it is now obvious that special relativity's effects such as the length variation cannot explain (as it was believed so far) these experiments since it is negligible. In the realm of mechanics, what would have happened if Newton was born after Einstein? We are exactly in this situation with respect to electromagnetism.

\section{Acknowledgement}

M.deM. is grateful to NSERC (Canada) for financial support. G.R. thanks E. Guyon, B. Jech, A. Domps, M. Le Bellac, O. Darrigol, R. Kofman, J. Rubin, J. Reignier and Y. Pierseaux for fruitful discussions on electromagnetism and relativity. G.R. was financially supported by a CNRS postdoctoral grant (S.P.M. section 02, France) during his stay in Nice. 


\section{References}

[1] Le Bellac M and Lévy-Leblond J M 1973 Galilean electromagnetism Nuov. Cim. B 14 217-233.

[2] Darrigol 02000 Electrodynamics from Ampère to Einstein (Oxford University Press)

[3] Crawford F S 1992 Magnetic monopoles, Galilean invariance, and Maxwell equations Am. J. Phys. 60 109-114;

Horzela A, Kapuścik E and Uzes C A 1993 Comment on "Magnetic monopoles, Galilean invariance, and Maxwell equations" Am. J. Phys. 61 471;

Crawford F S 1993 A response to A. Horzela, E. Kapuścik and C.A. Uzes' "Comment on 'Magnetic monopoles, Galilean invariance, and Maxwell equations'" Am. J. Phys. 61472.

[4] In this paper, we emphasize the Riemann-Lorenz approach to electromagnetism. Therein the central role is played by the vector and scalar potentials, unlike the Heaviside-Hertz approach, which rather relies on the fields themselves. For a justification, see Ref. [12] below.

[5] Goldstein H 1980 Classical Mechanics (Reading, Massachusetts: AddisonWesley).

[6] Lévy-Leblond J M 1965 Une nouvelle limite non-relativiste du groupe de Poincaré Ann. Inst. H. Poincaré 3 B 1-12.

[7] Bacry H and Lévy-Leblond J M 1968 Possible kinematics J. Math. Phys. 9 16051614.

[8] Cullwick E G 1957 Electromagnetism and Relativity (London: Longmans, Greenand);

Montgomery H 2004 Current flow patterns in a Faraday disc Eur. J. Phys. 25 171-183;

Montgomery H 1999 Unipolar induction: a neglected topic in the teaching of electromagnetism Eur. J. Phys. 20 271-280;

Jefimenko O D 1996 Is magnetic field due to an electric current a relativistic effect? Eur. J. Phys. 17 180-182;

Barnett S J 1912 On electromagnetic induction and relative motion Phys. Rev. 35 323-336;

Kholmetskii A L 2003 One century later: remarks on the Barnett experiment Am. J. Phys. 71 558-561; 
Redzic D V 2004 Conductors moving in magnetic fields: approach to equilibrium Eur. J. Phys. 25 623-632;

Redzic D V 2002 Electromagnetism of rotating conductors revisited Eur. J. Phys. 23 127-134;

Bringuier E 2003 Electrostatic charges in $\mathbf{v} \times \mathbf{B}$ fields and the phenomenon of induction Eur. J. Phys. 24 21-29;

Redzic D V 2004 Electrostatic charges in $\mathbf{~} \times \mathbf{B}$ fields: with or without special relativity? Eur. J. Phys. 25 L9-L11;

Bringuier E 2004 Reply to Redzic's Comment: Electrostatic charges in $\mathbf{v} \times \mathbf{B}$ fields without special relativity Eur. J. Phys. 25 L13-L15;

Lorrain P 2001 Electrostatic charges in $\mathbf{v} \times \mathbf{B}$ fields Eur. J. Phys. 22 L3-L4;

Lorrain P 1998 J. McTavish and F. Lorrain, Magnetic fields in moving conductors: four simple examples Eur. J. Phys. 19 451-457;

Kennard E H 1913 The effect of dielectrics on unipolar induction Phys. Rev. 1 355-359.

[9] Rousseaux G 2005 Lorenz or Coulomb in Galilean electromagnetism? Europhys. Lett. 71 15-20.

[10] Poincaré H 1890 (1st ed.) 1901 (2nd ed.) Electricité et Optique: Cours de Physique Mathématique, Paris: G. Carré (Book available at:

http : //gallica.bnf.fr/).

[11] Levi-Civita T 1897 Sulla reducibilità delle equazioni ellettrodinamiche di Helmholtz alla forme hertziana Nuov. Cim. VI (4) 93-108;

Levi-Civita T 1902 Sur le champ électromagnétique engendré par la translation uniforme d'une charge électrique parallèlement un plan conducteur indéfini $A n$ nales de la faculté des sciences de Toulouse, Sér. 24 5-44 (Article available at: http : //archive.numdam.org/article/AFST_1902_2_4_5_0.pdf).

[12] O'Rahilly A 1965 Electromagnetic Theory, a Critical Examination of Fundamentals (New York: Dover);

$\mathrm{Su}$ C C 2001 Explicit definitions of electric and magnetic fields in potentials and derivation of Maxwell's equations Eur. J. Phys. 22 L5-L8;

Yakubov V P 2004 Special theory of relativity in electrodynamics Russian Phys. J. 47 726-738;

Jefimenko O D 2004 Presenting electromagnetic theory in accordance with the principle of causality Eur. J. Phys. 25 287-296. 
[13] Dutch physicist Hendrik Anton Lorentz is often credited for the gauge condition and the formulation, whereas it is actually due to Danish physicist Ludvig Valentin Lorenz. For more details, see Jackson J D and Okun L B 2001 Historical roots of gauge invariance Rev. Mod. Phys. 73 663-680 and Keller O 1997 Optical works of L. V. Lorenz in Progress in Optics XXXVII, edited by E. Wolf (Amsterdam: North-Holland) 257343.

[14] Rousseaux G 2003 On the physical meaning of the gauge conditions of classical electrodynamics: the hydrodynamics analogue viewpoint Ann. Fondation Louis de Broglie 28 261-9. (Article available at:

http : //fr.arxiv.org/abs/physics/0511047).

[15] Fermi E. 1932 Quantum Theory of Radiation, Review of Modern Physics 4 87132 ;

Van Oosten A. 2000 A theory of electromagnetism with uniquely defined potentials and covariant conserved spin Eur. Phys. J. D 8 9-12.

[16] Santos E S, de Montigny M, Khanna F C and Santana A E, 2004 Galilean covariant Lagrangian models Ann. Phys. (NY) 37 9771-9789;

de Montigny M, Khanna F C and Santana A E, 2003 Nonrelativistic wave equations with gauge fields Int. J. Theor. Phys. 42 649-671.

[17] Takahashi Y 1988 Towards the many-body theory with the Galilei invariance as a guide. I. Fortschr. Phys. 36 63-81;

Takahashi Y 1988 Towards the many-body theory with the Galilei invariance as a guide. II. Fortschr. Phys. 36 83-96;

Omote M, Kamefuchi S, Takahashi Y and Ohnuki Y 1989 Galilean covariance and the Schrödinger equation Fortschr. Phys. 37 933-950.

[18] de Montigny M, Khanna F C, Santana A E, Santos E S and Vianna J D M 2000 Galilean covariance and the Duffin-Kemmer-Petiau equation J. Phys. A: Math. Gen. 33 L273-L278;

de Montigny M, Khanna F C, Santana A E and Santos E S 2001 Galilean covariance and the non-relativistic Bhabha equations J. Phys. A: Math. Gen. 34 8901-8917.

[19] Duval C, Burdet G, Künzle H P and Perrin M, 1985 Bargmann structures and Newton-Cartan theory Phys. Rev. D 31 1841-1853;

Künzle H P and Duval C 1994 Relativistic and nonrelativistic physical theories on five-dimensional space-time, in Semantical Aspects of Spacetime Theories Eds. U. Majer and H.J. Schmidt (Mannheim: BI-Wissenschaftsverlag) 113-129, and references therein; 
Kapuścik E 1986 On the physical meaning of the Galilean space-time coordinates Acta Phys. Pol. B 17 569-575.

[20] Maxwell J C 1954 A Treatise on Electricity and Magnetism, Vol. I and II (New York: Dover, 1st ed: Oxford, 1873)

[21] Melcher J R and Haus H A 1998 Electromagnetic Fields and Energy (Hypermedia Teaching Facility, M.I.T.) (Available at: http : //web.mit.edu/6.013_book/www/);

Zhan M and Haus H A 1995 Contribution of Prof. James R. Melcher to engineering education J. Electrostatics 34 109;

Moreau R 1990 Magnetohydrodynamics (Amsterdam: Kluwer Academic);

Castellanos A Electrohydrodynamics Lecture Notes of the 7th IUTAM Summer School, Udine, 22-26 July, 1996.

[22] Earman J 1989 World Enough and Space-Time, Absolute versus Relational Theories of Space and Time (Cambridge, Mass.: MIT);

Rynasiewicz R 2003 Field unification in the Maxwell-Lorentz theory with absolute space Phil. Sci. 70 1063-1072. (Article available at: http ://philsci - archive.pitt.edu/archive/00001096/index.html)

[23] Dyson F J 1990 Feynman's proof of the Maxwell equations Am. J. Phys. 58 209-211.

[24] Brown H R and Holland P R 1999 The Galilean covariance of quantum mechanics in the case of external fields Am. J. Phys. 67 204-214.

[25] Vaidya A and Farina C 1991 Can Galilean mechanics and full Maxwell equations coexist peacefully? Phys. Lett. A 153 265-267.

[26] Brown H R and Holland P R 2003 The non-relativistic limit of the Maxwell and Dirac equations: The role of Galilean and gauge invariance Studies in the History and Philosophy of Modern Physics 34 161-87.

[27] Lévy-Leblond J M 1967 Nonrelativistic particles and wave equations Comm. Math. Phys. 6 286-311.

[28] Greiter M 2005 Is electromagnetic gauge invariance spontaneously violated in superconductors? Ann. Phys. (NY) 319 217-249.

[29] Panofsky W K H and Phillips M 1955 Classical Electricity and Magnetism (New York: Adison-Wesley).

[30] Drezet A 2005 The physical origin of the Fresnel drag of light by a moving dielectric medium Eur. Phys. J. B 45 103-110. 
[31] Lorentz H A 1904 Remarque au sujet de l'induction unipolaire Arch. Néer. 9 (1904) 380.

[32] Minkowski H 1908 Die Grundleichungen fur die elektromagnetischen Vorgange in bewegten Koerpern Nachr. Ges. Wiss. Gottingen 53 ; 1910 Math. Annalen 68 472;

Arzeliès H 1959 Milieux conducteurs ou polarisables en mouvement, Etudes Relativistes (Paris: Gauthier-Villars).

[33] Einstein A and Laub J 1908 Uber die elektromagnetischen Grundgleichungen fur bewegte Koerper Annalen Phys. 26 532-539;

Einstein A and Laub J 1908 Uber die im elektromagnetischen Felde auf ruhende Koerper ausgebten ponderomotorischen Krafte Annalen Phys. 26 541-550.

[34] Roentgen 1888 Uber die durch Bewegung eines im homogenen elektrischen Felde befindlichen Dielectricums hervorgerufene elektrodynamische Kraft Annalen Phys. 35264.

[35] Eichenwald A 1903 Uber die magnetischen Wirkungen bewegter Koerper im elektrostatischen Felde Annalen Phys. 11 1-30.

[36] Wilson H A 1904 On the electric effect of rotating a dielectric in a magnetic field Phil. Trans. Roy. Soc. London 204 121-137.

[37] Wilson M and Wilson H A 1913 On the electric effect of rotating a magnetic insulator in a magnetic field Phil. Trans. Roy. Soc. London 89 99-106;

Hertzberg J B, Bickman S R, Hummon M T, Krause D, Peck S K and Hunter L R 2001 Measurement of the relativistic potential difference across a rotating dielectric cylinder Am. J. Phys. 69 648-654.

[38] Trouton F T 1902 The results of an electrical experiment involving the relative motion of the earth and ether Transactions of the Royal Dublin Society 7 379-384;

Trouton F T and Noble H R 1903 The mechanical forces acting on a charged electric condenser moving through space Phil. Trans. R. Soc. A 202 165-181;

Hayden H C 1994 High sensitivity Trouton-Noble experiment Rev. Sc. Instrum. 65 788-792;

Jefimenko O D 1999 The Trouton-Noble paradox J. Phys. A: Math. Gen. 32 3755-3762.

[39] Darrigol O 1993 The electrodynamics of moving bodies from Faraday to Hertz Centaurus 36 245-260;

Darrigol O 1995 Emil Cohn's electrodynamics of moving bodies Am. J. Phys. 63 908-915. 
[40] Einstein A 1905 Zur Elektrodynamik bewegter Korper Annalen Phys. 17 891; Miller A I 1981 Albert Einstein's Special Theory of Relativity (New York: Addison-Wesley).

[41] Norton J D 2004 Einstein's investigation of Galilean covariant electrodynamics prior to 1905 Archive for History of Exact Sciences 59 45-105;

Hon G and Goldstein B R 2005 How Einstein made asymmetry disappear: symmetry and relativity in 1905 Archive for History of Exact Sciences 59 437-544.

[42] Pauli W 2000 Electrodynamics, Pauli Lectures on Physics Volume 1 (New York: Dover) 106-108.

[43] Keswani G H and Kilmister C W 1983 Intimations of relativity: relativity before Einstein Brit. F. Phil. Sci. 34 343-354. 